\title{
Effects of community-based exercise in adults with severe burns: a randomized controlled trial
}

\author{
Charles D. Voigt, M.D. ${ }^{a}$, Guillermo Foncerrada, M.D. ${ }^{b, c}$, Raquel Peña, M.D. ${ }^{b, c}$, Ashley N. \\ Guillory, Ph.D. ${ }^{b, c}$, Clark R. Andersen, M.S. ${ }^{d}$, Craig G. Crandall, Ph.D. ${ }^{f}$, Steven E. Wolf, M.D. ${ }^{e}$, \\ David N. Herndon, M.D. ${ }^{\text {b,c }}$, Oscar E. Suman, Ph.D. ${ }^{\text {b,c }}$ \\ aDepartment of Surgery, Creighton University, Omaha, NE, United States \\ bDepartment of Surgery, University of Texas Medical Branch, Galveston, TX, United States \\ 'Shriners Hospitals for Children, Galveston, TX, United States \\ ${ }^{\mathrm{d} D e p a r t m e n t}$ of Preventative Medicine and Community Health, University of Texas Medical \\ Branch, Galveston, TX, United States \\ eInstitute for Exercise and Environmental Medicine, Texas Health Presbyterian Hospital Dallas \\ and University of Texas Southwestern Medical Center, Dallas, TX, United States \\ fDivision of Burns, Trauma, and Critical Care, Department of Surgery, University of Texas \\ Southwestern, Dallas, TX, United States
}

\section{Abstract \\ Objective: To investigate the efficacy of community-based exercise programs (COMBEX) in the rehabilitation of adult burn patients as compared to standard of care (SOC).}

Design: Randomized controlled trial, with 2:1 randomization.

Setting: Assessments were performed in a hospital setting. The intervention was performed in a community setting.

Participants: Adult patients with $\geq 30 \%$ total body surface area burns were randomized to participate in COMBEX $(\mathrm{N}=31)$ or standard of care (SOC) $(\mathrm{N}=14)$. Patient sampling was consecutive and referred.

\footnotetext{
Corresponding author: Oscar E. Suman, 815 Market Street, Galveston Texas 77550, (409)-770-6557, oesuman@utmb.edu. Publisher's Disclaimer: This is a PDF file of an unedited manuscript that has been accepted for publication. As a service to our customers we are providing this early version of the manuscript. The manuscript will undergo copyediting, typesetting, and review of the resulting proof before it is published in its final citable form. Please note that during the production process errors may be discovered which could affect the content, and all legal disclaimers that apply to the journal pertain.

The authors declare that there are no conflicts of interest or financial affiliations specific to this study.

This study is registered at clinicaltrials.gov (\#NCT01184547).

Authors' contributions

Drs. Suman, Crandall, Wolf, and Herndon are responsible for the conception and design of the study. Dr. Voigt and Clark Andersen analyzed data. All authors contributed to the interpretation of results and drafting of the manuscript. Drs. Voigt and Suman prepared the figures. All authors approved the final version of the manuscript.

Suppliers List
} 
Interventions: The COMBEX program consisted of 12 weeks of exercise with a communitybased trainer after hospital discharge. The SOC group did not receive exercise training.

Main Outcome Measures: Change in lean body mass index, peak torque, and VO2 max from discharge to 12 weeks post discharge, presented as mean \pm SE.

Results: The COMBEX group showed a significant increase in VO2 max compared to SOC (COMBEX: $\Delta=7.723 \pm 1.522 \mathrm{~mL} / \mathrm{kg} / \mathrm{min}, \mathrm{p}=0.0006$; SOC: $\Delta=2.200 \pm 1.150 \mathrm{~mL} / \mathrm{kg} / \mathrm{min}, \mathrm{p}=$ 0.0765; COMBEX vs SOC, $\mathrm{p}=0.0236$ ). The COMBEX group exhibited a significant within group increase in lean body mass index $\left(\Delta=1.107 \pm 0.431 \mathrm{~kg} / \mathrm{m}^{2}, \mathrm{p}=0.0003\right.$; SOC: $\Delta=1.323 \pm$ $\left.0.873 \mathrm{~kg} / \mathrm{m}^{2}, \mathrm{p}=0.2808\right)$. Both groups showed significant within group increases in peak torque (COMBEX: $\Delta=35.645 \pm 7.566 \mathrm{~N} \mathrm{~m}, \mathrm{p}=0.0003$; SOC: $\Delta=34.717 \pm 11.029 \mathrm{~N} \mathrm{~m}, \mathrm{p}=0.0082$ ). No significant differences were noted between the 2 groups for LBMI or peak torque.

Conclusions: Patients who participate in a community-based exercise program show significant improvements in cardiopulmonary fitness compared to standard of care, supporting the use of COMBEX as an alternative therapy to SOC in adults with severe burns.

\section{Keywords}

Physical Fitness; Torque; Oxygen Consumption; Body Composition

Severe burn injury has been shown to result in profound morbidity owing to hypermetabolic and hypercatabolic responses that can last for at least 24 months following injury.(1) These responses lead to loss of lean body mass, (2) which is exacerbated by reduced physical activity as a result of the injury.(3) Our institution has demonstrated that 12-week in-hospital exercise programs can improve lean body mass, muscle strength and power, and maximal aerobic capacity in burned children.(4-6) However, incorporating a hospital-based exercise program into the post-discharge care of the burn patient has advantages and disadvantages that must be weighed before implementation. Exercise programs carried out in hospitals that treat burn injuries will have staff that is specialized or familiar with the requirements of these patients. However, the distance from the patient's home requires significant time commitments and monetary resources for participation. These disadvantages of participating in-hospital exercise programs are compounded in adult burn patients because of family/ personal commitments and the need of many to return to work. Thus, many patients opt to forgo these structured exercise programs. A recent study of exercise behaviors in adult burn survivors suggests that those with burn injury are less likely to engage in regularly scheduled exercise because of their physical condition or lack of motivation.(7)

To address this issue, we have investigated the use of a community-based exercise program (COMBEX) in the rehabilitation of pediatric burn patients. We have shown that such programs are viable alternatives to hospital-based exercise programs in improving lean body mass, muscle strength, and cardiopulmonary fitness.(8) The objective of this study is to extend our previous work in the pediatric burn population into the adult burn population. We hypothesized that adult burn patients who underwent community-based exercise programs would have improvements in body composition and measures of physical and cardiopulmonary fitness as compared to those who did not. 


\section{Methods}

This was a multi-center, prospective, randomized study, which conforms to the Helsinki Declaration and was approved by the Institutional Review Board. Outside institutions, consisting of other regional burn units which provide the same level of care, referred patients to the primary institution for testing. Costs of travel to the primary institution for testing was covered. Patients were enrolled from November 2009 through January, 2016. All patients were adults who agreed to participate in the study and signed an informed consent form, which was approved by the Institutional Review Board. A power analysis was performed with an alpha of 0.01 to obtain a family error rate of less than 0.05 , and a beta of 0.8 . A sample size of 20 patients per group was determined to be able to detect a difference in change in muscle mass of $5 \%$, change in aerobic capacity of $20 \%$, and change in muscle strength of $13 \%$. After providing consent, patients were randomized to either COMBEX (N $=31)$ or standard of care $(\mathrm{SOC})(\mathrm{N}=14)$ in a 2:1 ratio. The prospective randomized schedule was created by the American Burn Association Data Coordinating Center. Inclusion and exclusion criteria are listed in Text Box 1. Endpoints of the study included lean body mass, muscle strength, and cardiopulmonary fitness, which were assessed at hospital discharge and at 12 weeks after exercise training. Assessors were not blinded during outcome measurements. All patients received SOC treatment during their hospitalization.

One week prior to discharge the patient was approached, and the study was explained to the patient. Participation was then offered. An eligibility checklist was filled out, and if qualified, patients were given a copy of the consent form for their review. The research personnel followed up with the patient the next day, and if the patient agreed to participate, a signature was obtained and a copy of signed consent left for their review and reference. The patient was then randomized into either of the groups according to the randomization table. A chart was made identifying the participant. Participants underwent outcome testing as described below upon discharge from the hospital, and were scheduled for a follow-up visit in 12 weeks for further outcome testing. Transportation for outcome testing was paid for by the institution.

\section{Standard of care group}

Upon discharge from the hospital, patients in the SOC group were given instructions on how to perform physical therapy and occupational therapy exercises after discharge. After education, patients could perform the physical therapy and occupational therapy exercises either at their own home or a gym, though there was no structured intervention or gym access provided to this arm. Rehabilitation therapy exercises that our institution educates patients upon varies depending upon individual needs, but examples of common techniques include compression therapy, hand therapy, activities of daily living, face and neck stretching, scar massages, splinting, range of motion and strength exercises, and restoration of function exercises.(9) 


\section{Community-based exercise program group}

Patients in the COMBEX group were also provided with instructions on how to perform physical therapy and occupational therapy exercises on their own. In addition, they were supplied with a pre-paid 12-week membership at an exercise facility near their home, as well as 36 pre-paid personal trainer sessions (to be attended 3 times per week), and they were prescribed a 12-week exercise training program. Transporation to gyms was not provided to participants. During these sessions, which made up the 12-week exercise training program, personal trainers were given a list of recommended aerobic and resistance training exercises. The personal trainers could modify the workout at their discretion based upon the needs of the participants. The aerobic exercise sessions consisted of warm-up, exercise, and cooldown phases performed for 20 to 40 minutes using a treadmill, elliptical, rower, or cycle ergometer per the patient's individual needs. Exercises were performed at an intensity to elicit 60 to $85 \%$ of the patient's peak heart rate or a rating of perceived exertion of 6 to 8 . (10) Resistive exercise sessions used variable resistance machines or free weights, with 30 minutes of exercises consisting of 3 sets of 8 to 15 repetitions. Progressive resistance training was carried out with a mixture of free weights and weightlifting machines. The types of exercises done were bench press, biceps and triceps curls, shoulder press, leg press, leg extension, hamstring curls, abdominal crunches. Progression of exercise loads, repetitions and sets were determined as previously described(8). Both groups received similar SOC treatment, which includes compression therapy and splinting if prescribed by burn surgeon with assistance from PT/OT. Patients were also free to exercise at the gym outside of the personal training sessions if they desired.

\section{Lean body mass index}

Body composition was assessed via dual energy X-ray absorptiometry ${ }^{\mathrm{a}}$ with patients in the supine position. Lean body mass index (LBMI) was reported in kilograms of body weight divided by the square of the patient's height in meters $\left(\mathrm{kg} / \mathrm{m}^{2}\right)$. The methodology used by our group has been reported in previous studies. These measurements were performed at hospital discharge and after completion of 12 weeks of exercise in the COMBEX group or at 12 weeks post discharge in the SOC group.

\section{Cardiopulmonary fitness}

Maximal aerobic capacity was assessed according to a modified Bruce protocol.(12) The incline and/or speed of the treadmill was adjusted every 3 minutes during the test. The initial speed of the treadmill was $1.7 \mathrm{mph}$, and the initial incline was zero.(12) The exercise intensity was progressively increased until the patient could not maintain the required workload (volitional fatigue). Other indicators of maximal or peak exertion included a respiratory quotient greater than 1.10 and a Borg's Rated Perceived Exertion level (CR-10) of 9-10. $(6,13)$ Methodologies for measurement and analysis of heart rate and $\mathrm{VO}_{2}$ peak $\left(\mathrm{mL} \mathrm{O} \mathrm{O}_{2}\right.$ consumed $/ \mathrm{kg}$ body weight $/ \mathrm{min}$ ) are described in our other work.(12) Peak heart rate and peak $\mathrm{VO}_{2}$ were used to guide the intensity of training of aerobic exercise in the

aHologic Inc, 35 Crosby Dr, Bedford, MA 01730 
COMBEX group. Similar to dual energy X-ray absorptiometry measurements, assessments were performed at hospital discharge and 12 weeks later.

\section{Muscle strength}

Peak torque was assessed at hospital discharge and 12 weeks later via an isokinetic dynamometer $^{\mathrm{b}}$, with peak torque being defined as the highest muscular force output (Biodex Advantage Software Operations Manual. Shirley, NY, 2001). Prior to testing, patients were told what to expect throughout the testing process. Patients were tested in a seated position with a strap over the midthigh and pelvis to keep them secure. Dominant leg extensors were tested at 150 degrees per second. Prior to testing, patients were allowed to complete a practice session of 10 repetitions at 150 degrees per second. During testing, patients performed 10 repetitions at 150 degrees per second at a maximal volitional effort.

\section{Statistical analyses}

For each outcome (VO2 maximum, peak torque, and lean body mass index), the change between discharge and post-exercise measurements was modeled by analysis of variance with relation to treatment group. Heterogeneous variance was controlled by weighting, and different mean baseline measurements at discharge for each group were controlled for via inclusion as a covariate in the statistical model. Multiple testing associated with reporting of main effects and treatment effects were controlled by Hommel-adjusted p-values. (14) Chisquare tests and Student's T tests were used to assess demographic differences between groups. All modeling was performed using R statistical software. (15) In all statistical tests, alpha $=.05$, for a $95 \%$ level of confidence. Results are presented as mean \pm SE.

\section{Results}

A total of 45 subjects were randomized to the COMBEX or SOC groups. Study subject disposition is outlined in the participant flow [CONSORT] diagram provided in Fig. 1. Demographic and other characteristics including age, sex, total body surface area burned, total body surface area with third-degree burns, and length of stay did not significantly differ between the COMBEX and SOC groups ( $p>0.05$, Table 1).

Measurements for LBMI, muscle strength, and cardiorespiratory fitness are presented in Table 2, along with the change in these measurements $(\Delta)$ and the within-group and between-group tests. Within-group comparisons showed that 12 weeks of exercise increased LBMI $\left(\Delta=1.107 \pm 0.431 \mathrm{~kg} / \mathrm{m}^{2}, p=0.0003\right)$ and $\mathrm{VO}_{2}$ peak $(\Delta=7.723 \pm 1.522 \mathrm{~mL} / \mathrm{kg} / \mathrm{min}$, $p=0.0006)$. No significant increases were detected in the SOC group for either LBMI $(\Delta=$ $\left.1.323 \pm 0.873 \mathrm{~kg} / \mathrm{m}^{2}, p=0.2808\right)$ or $\mathrm{VO}_{2}$ peak $(\Delta=2.200 \pm 1.150 \mathrm{~mL} / \mathrm{kg} / \mathrm{min}, p=0.0765)$. In contrast, both the COMBEX and SOC groups had significant within-group increases in peak torque muscle strength (COMBEX: $\Delta=35.645 \pm 7.566 \mathrm{~N} \mathrm{~m}, p=0.0003$; SOC: $\Delta=$ $34.717 \pm 11.029 \mathrm{~N} \mathrm{~m}, p=0.0082$ ). Between group comparison showed a significant increase in $\mathrm{VO}_{2}$ peak in the COMBEX group as compared to the $\mathrm{SOC}$ group (SOC-COMBEX =

\footnotetext{
biodex Medical Systems, Inc, 20 Ramsey Rd, Shirley, NY 11967
} 
$5.523 \pm 1.908 \mathrm{~mL} / \mathrm{kg} / \mathrm{min}, p=0.0236$ ). However, between-group comparisons showed no significant differences in LBMI or peak torque muscle strength between the COMBEX and SOC groups.

\section{Discussion}

This study shows that adults who engage in a 12-week community-based exercise program following burn injury experience improvements in lean body mass index, muscle strength, and cardiopulmonary capacity. Of note, the patients who underwent community-based exercise programs had a significant improvement in their cardiopulmonary capacity, as measured by $\mathrm{VO}_{2}$ peak, when compared to those who did note engage in a guided exercise program. The improvements in lean body mass index and muscle strength were significant by within-group comparison, but not when compared to the SOC group. The increase in $\mathrm{VO}_{2}$ peak, representing increased exercise tolerance, has been noted not only with burned children, but also in other disease processes including COPD. (17) These results agree with those of previous studies from our institution investigating both in-hospital and communitybased exercise programs in the pediatric burn population(4-6, 8, 13, 18-21), as well as in studies done by others in adult burn patients. Ebid, et. al., noted that 12 weeks of isokinetic exercise training in burned adults resulted in increased strength, though not increased to the level of a healthy adult (22), with similar results in children. (23)While in-hospital exercise programs are offered to all of our pediatric patients as SOC, these programs have yet to be implemented in our adult patients owing to lack of studies in the burned adult population as well as barriers to participating in an in-hospital exercise program such as cost, time, distance from home, and loss of work.

While the patients who were enrolled in COMBEX had improvements in all 3 measures studied, those who were in the SOC group showed significant improvements in only peak torque muscle strength. We hypothesize that this improvement in strength in the SOC group is primarily due to early neuromuscular adaptations.(24) Those in the SOC group experienced a significant increase in muscle activity from the transition from hospitalization to day-to-day activities, which included unguided physical therapy/occupational therapy exercises supplied to each patient. This increase in activity necessitated improved motor unit activation and subsequently increased strength without corresponding muscle hypertrophy. However, these exercises were only suggested to patients, and without guidance, patients may have significant issues with compliance. In contrast, structured exercise improved both muscle strength and LBMI, showing that muscle hypertrophy took place. The proportion of the strength increase representing neural gains and that attributable to hypertrophy was not determined in this study, though future studies of longer exercise programs could show greater improvements in exercise outcomes.

Our results suggest that community-based exercise programs may be efficacious when included as part of post-burn rehabilitation. Moving forward, studies should elaborate on the advantages of taking part in these programs as well as determine ways to individualize exercise programs to the needs of the burn patient. While community gyms and personal trainers may have less experience with the specific needs of this population, improved relationships between burn care providers and personal trainers in the region may promote 
participation in such programs. They may also promote education of personal trainers so that these community programs will more closely mirror their hospital-based counterparts.

Future studies should also focus on psychosocial benefits of exercise training using personal trainers and community gyms compared to hospital based exercise training programs. There is a possibility that exercise training in the community gym using personal exercise trainers could improve self-confidence and promote a quicker reintegration into society.

\section{Study limitations}

A limitation of the study is the small sample size. This, taken with variation among subjects, may account for lack of differences detected between the COMBEX and SOC groups. Future studies with greater power will strengthen these results. The small sample size was compounded by lack of compliance and incomplete numbers of measurements at each time point. This was especially true in the SOC group. The lack of an intervention in the SOC group likely led to more missed follow-up appointments, reducing the numbers of measurements. Future studies should incorporate improved methods of incentivizing nonintervention patients to return for follow-up measurements. Compliance for the personal training sessions in the COMBEX group was to be communicated to our institution via attendance logs requested from the personal trainers or the community-based gyms.

However, these were not provided for most of the patients. Future studies of communitybased interventions should incorporate improved methods of communication with patients and personal trainers during exercise programs.

\section{Conclusions}

Adult burn patients who participate in a 12-week COMBEX program show significant improvement in cardiopulmonary capacity as compared to those who do not engage in a structured exercise program.. These results support the integration of COMBEX programs into post-burn rehabilitation.

\section{Acknowledgments}

The authors would like to acknowledge all clinical and scientific staff at Shriners Hospitals for Children ${ }^{\circledR}$ _ Galveston for their roles in patient care and scientific research. In addition, we thank Dr. Kasie Cole for editing support. We thank those at University of Pittsburgh for recruiting 2 patients, the University of Texas Southwestern for recruiting 5 patients, the United States Army Institute of Surgical Research for recruiting 1 patient, and Memorial Herman Hospital for recruiting 2 patients.

This study was partially funded by grants from the following sources: NIH (P50-GM060388, R01-HD049471, R01HD049471-08S1, T32-GM008256), NIDILRR (90DP0043-01-00), Department of Defense/American Burn Association (W81XWH-09-2-0194), and Shriners Hospitals for Children $(84080,84291)$. None of the sponsors had any role in the study design; in the collection, analysis, or interpretation of data; in the writing of the report; or in the decision to submit the article for publication.

\section{List of Abbreviations:}

COMBEX community-based exercise program

LBMI lean body mass index

MPH miles per hour 
standard of care

$\mathrm{VO}_{2 \text { peak }}$

maximum oxygen uptake

\section{References}

1. Jeschke MG, Chinkes DL, Finnerty CC, Kulp G, Suman OE, Norbury WB, et al. Pathophysiologic response to severe burn injury. Ann Surg. 2008;248(3):387-400. [PubMed: 18791359]

2. Hart DW, Wolf SE, Chinkes DL, Gore DC, Mlcak RP, Beauford RB, et al. Determinants of skeletal muscle catabolism after severe burn. Ann Surg. 2000;232(4):455-65. [PubMed: 10998644]

3. Ferrando AA, Tipton KD, Bamman MM, Wolfe RR. Resistance exercise maintains skeletal muscle protein synthesis during bed rest. J Appl Physiol (1985). 1997;82(3):807-10. [PubMed: 9074967]

4. Suman OE, Herndon DN. Effects of cessation of a structured and supervised exercise conditioning program on lean mass and muscle strength in severely burned children. Arch Phys Med Rehabil. 2007;88(12 Suppl 2):S24-9. [PubMed: 18036977]

5. Celis MM, Suman OE, Huang TT, Yen P, Herndon DN. Effect of a supervised exercise and physiotherapy program on surgical interventions in children with thermal injury. J Burn Care Rehabil. 2003;24(1):57-61; discussion 56. [PubMed: 12543995]

6. Suman OE, Spies RJ, Celis MM, Mlcak RP, Herndon DN. Effects of a 12-wk resistance exercise program on skeletal muscle strength in children with burn injuries. J Appl Physiol (1985). 2001;91(3):1168-75. [PubMed: 11509512]

7. Baldwin J, Li F. Exercise behaviors after burn injury. J Burn Care Res. 2013;34(5):529-36. [PubMed: 23816997]

8. Pena R, Ramirez LL, Crandall CG, Wolf SE, Herndon DN, Suman OE. Effects of community-based exercise in children with severe burns: A randomized trial. Burns. 2016;42(1):41-7. [PubMed: 26643401]

9. Serghiou MA, Ott S, Whitehead C, Cowan A, McEntire S, Suman OE. Comprehensive rehabilitation of the burn patient In: Herndon DN, editor. Total Burn Care. 4th ed. Philadelphia, PA: Elsevier; 2012 p. 517-49.

10. Garber CE, Blissmer B, Deschenes MR, Franklin BA, Lamonte MJ, Lee IM, et al. American College of Sports Medicine position stand. Quantity and quality of exercise for developing and maintaining cardiorespiratory, musculoskeletal, and neuromotor fitness in apparently healthy adults: guidance for prescribing exercise. Med Sci Sports Exerc. 2011;43(7):1334-59. [PubMed: 21694556]

11. Wurzer P, Voigt CD, Clayton RP, Andersen CR, Mlcak RP, Kamolz LP, et al. Long-term effects of physical exercise during rehabilitation in patients with severe burns. Surgery. 2016;160(3):781-8. [PubMed: 27267551]

12. Jones N. Clinical exercise testing. 4th ed. Philadelphia, PA: W.B. Saunders Company; 1997.

13. Przkora R, Herndon DN, Suman OE. The effects of oxandrolone and exercise on muscle mass and function in children with severe burns. Pediatrics. 2007;119(1):e109-16. [PubMed: 17130281]

14. Hommel G. A stagewise rejective multiple test procedure based on a modified Bonferroni test. Biometrika. 1988;75:383-6.

15. Team RC. R: A language and environment for statistical computing. Vienna, Austria: R Foundation for Statistical Computing; 2017.

16. Team RC. R: A language and environment for statistical computing. Vienna, Austria: R Foundation for Statisitical Computing; 2016.

17. Suman OE, Mlcak RP, Herndon DN. Effect of exercise training on pulmonary function in children with thermal injury. J Burn Care Rehabil. 2002;23(4):288-93; discussion 7. [PubMed: 12142585]

18. Suman OE, Thomas SJ, Wilkins JP, Mlcak RP, Herndon DN. Effect of exogenous growth hormone and exercise on lean mass and muscle function in children with burns. J Appl Physiol (1985). 2003;94(6):2273-81. [PubMed: 12588788] 
19. Neugebauer CT, Serghiou M, Herndon DN, Suman OE. Effects of a 12-week rehabilitation program with music \& exercise groups on range of motion in young children with severe burns. J Burn Care Res. 2008;29(6):939-48. [PubMed: 18849852]

20. Rosenberg M, Celis MM, Meyer W 3rd, Tropez-Arceneaux L, McEntire SJ, Fuchs H, et al. Effects of a hospital based Wellness and Exercise program on quality of life of children with severe burns. Burns. 2013;39(4):599-609. [PubMed: 22985974]

21. Porro LJ, Al-Mousawi AM, Williams F, Herndon DN, Mlcak RP, Suman OE. Effects of Propranolol and Exercise Training in Children with Severe Burns. Journal of Pediatrics. 2013;162(4):799-+. [PubMed: 23084706]

22. Ebid AA, Omar MT, Abd El Baky AM. Effect of 12-week isokinetic training on muscle strength in adult with healed thermal burn. Burns. 2012;38(1):61-8. [PubMed: 22103985]

23. Ebid AA, El-Shamy SM, Draz AH. Effect of isokinetic training on muscle strength, size and gait after healed pediatric burn: a randomized controlled study. Burns. 2014;40(1):97-105. [PubMed: 24074720]

24. Gabriel DA, Kamen G, Frost G. Neural adaptations to resistive exercise: mechanisms and recommendations for training practices. Sports Med. 2006;36(2):133-49. [PubMed: 16464122] 


\section{Text Box 1 Inclusion and Exclusion Criteria}

\section{Inclusion Criteria}

- Total body surface area burned $\geq 30 \%$

- $\quad$ Age $\geq 18$ years and $\leq 59$ years

\section{Exclusion Criteria}

- Known history of AIDS, AIDS Related Complex, or HIV

- Malignant neuroleptic hyperthermia

- $\quad$ Active tuberculosis

- $\quad$ Cirrhosis

- $\quad$ Renal insufficiency

- Hepatic disease

- Known coronary artery disease

- Congestive heart failure

- Uncontrolled asthma or pulmonary disease

- Associated head injuries requiring specific treatment

- Mental retardation/autism/other mental disorder

- Gastrointestinal disorders that impair absorption

- $\quad$ Pregnancy 


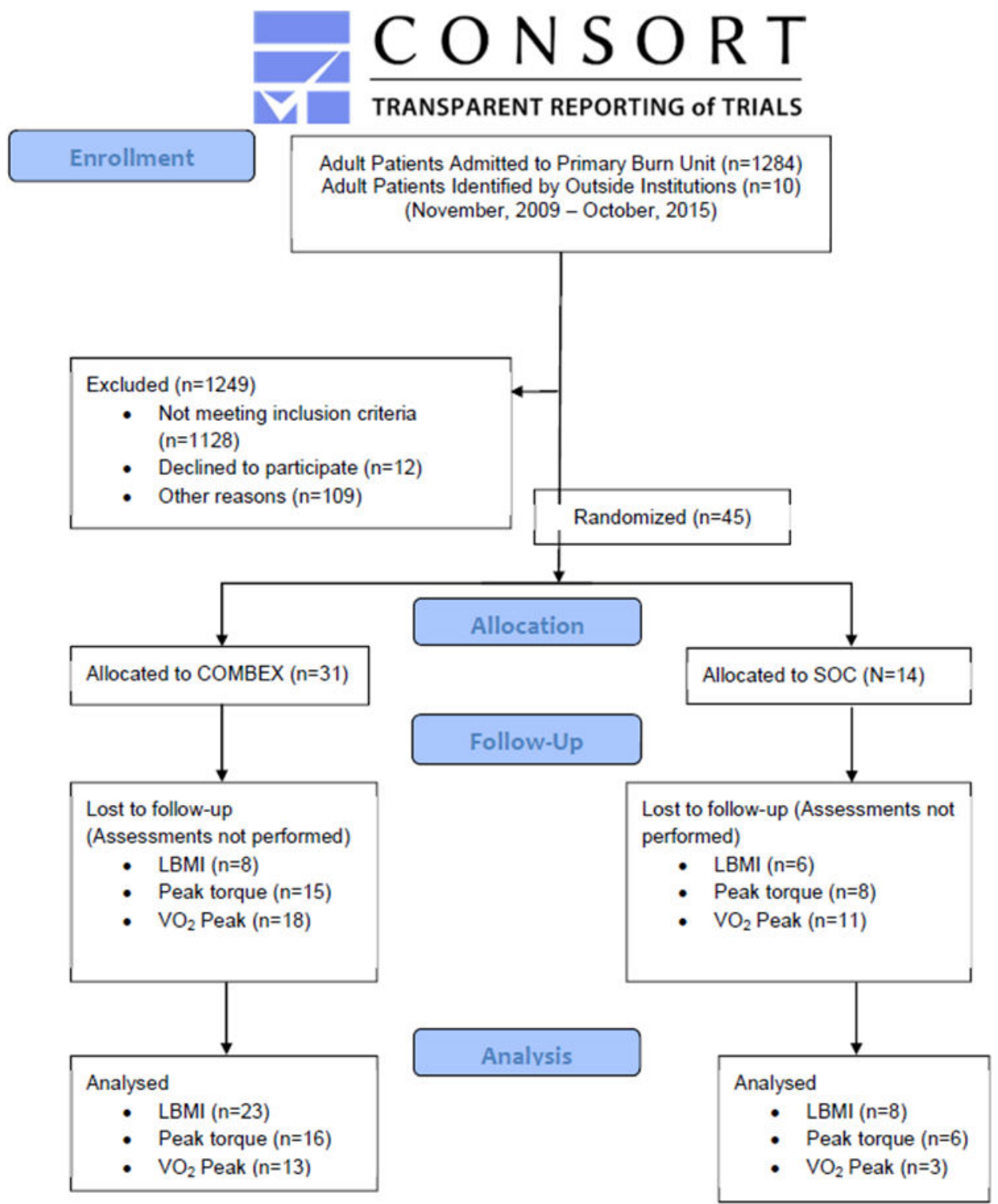

Figure 1:

CONSORT flow diagram for the study. 


\section{Table 1}

Patient Characteristics

\begin{tabular}{llll}
\hline Characteristic & \multicolumn{3}{c}{ Group } \\
& COMBEX & SOC & $\boldsymbol{p}$ value \\
\hline $\boldsymbol{N}$ & 31 & 14 & \\
Sex (Male/Female) & $30 / 1$ & $14 / 0$ & 1.00 \\
Age (Years) & $35 \pm 11$ & $35 \pm 9$ & 0.86 \\
TBSA Burned (\%) & $46 \pm 11 \%$ & $48 \pm 19 \%$ & 0.73 \\
TBSA Burned, 3rd-Degree (\%) & $25 \pm 18 \%$ & $37 \pm 22 \%$ & 0.18 \\
LOS (days) & $41 \pm 34$ & $44 \pm 40$ & 0.83 \\
\hline
\end{tabular}

Values are presented as mean \pm SD. COMBEX (community-based exercise program), SOC (standard of care), TBSA (total body surface area), LOS (length of stay). 


\section{Table 2}

Lean Body Mass Index, Peak Torque, and $\mathrm{VO}_{2}$ Peak

\begin{tabular}{|l|c|c|c|c|c|}
\hline Measure & \multicolumn{2}{|c|}{$\boldsymbol{\Delta}$ from DC to Post-Exercise, COMBEX } & $\boldsymbol{\Delta}$ from DC to Post-Exercise, SOC & COMBEX-SOC \\
\hline & Mean \pm SE & $p$-value & Mean \pm SE & $p$-value & $p$-value \\
\hline VO $_{\mathbf{2}}$ Peak $(\mathbf{m L} / \mathbf{k g} / \mathbf{m i n})$ & $7.723 \pm 1.522$ & 0.0006 & $2.200 \pm 0.0765$ & 0.0765 & 0.0236 \\
\hline Peak torque $(\mathbf{N} \mathbf{~ m})$ & $35.645 \pm 7.566$ & 0.0003 & $34.717 \pm 11.029$ & 0.0082 & 0.9452 \\
\hline Lean Body Mass Index $\left(\mathbf{k g} / \mathbf{m}^{\mathbf{2}}\right)$ & $1.107 \pm 0.431$ & 0.0468 & $1.323 \pm 0.873$ & 0.2808 & 0.8259 \\
\hline
\end{tabular}

Values are expressed as mean \pm SED. COMBEX (community-based exercise program), SOC (standard of care). $P$-values are Hommel adjusted. 